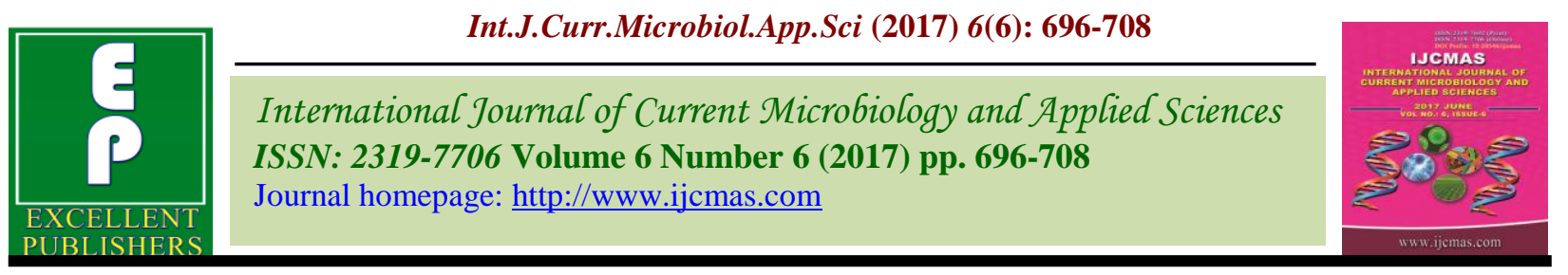

Original Research Article https://doi.org/10.20546/ijcmas.2017.606.082

\title{
Effect of Natural Enemies on the Population Dynamics of Insect-Pest of Cabbage Ecosystem
}

\section{G.C. Jat ${ }^{1}$, R. Swaminathan ${ }^{2}$, P.C. Yadav $^{1}$, H.L. Deshwal ${ }^{3}$, S.K. Dotasara ${ }^{4}$, Suman Choudhary ${ }^{4}$ and Hari Singh Choudhary ${ }^{4}$}

${ }^{1}$ Department of Entomology, Rajasthan College of Agriculture, (MPUAT) Udaipur, Rajasthan, India

${ }^{2}$ Dean, Rajasthan College of Agriculture, (MPUAT) Udaipur, Rajasthan, India ${ }^{3}$ Department of Entomology, College of Agriculture and Agriculture Research Station, (SKRAU) Bikaner, Rajasthan, India

${ }^{4}$ Department of Entomology, SKNCOA (SKNAU) Jobner, Jaipur, Rajasthan, India *Corresponding author

\begin{tabular}{|c|c|}
\hline & A B S T R A C T \\
\hline & Field experiments were conducted during Rabi season of 2012-13 and \\
\hline & $\begin{array}{l}\text { 2013-14. The study revealed that the major natural enemies recorded were } \\
\text { coccinellids, Coccinella septempunctata (Fab.). Cheilomenes sexmaculatus }\end{array}$ \\
\hline $\begin{array}{l}\text { Insect pests, } \\
\text { Natural enemies, }\end{array}$ & (Fab.) (Coleoptera: Coccinellidae); common green lacewing, Chrysoperla \\
\hline $\begin{array}{l}\text { Cabbage and } \\
\text { Percent parasitism. }\end{array}$ & $\begin{array}{l}\text { sp. (Neuroptera: Chrysopidae); aphid parasitoid, Diaeretiella sp. } \\
\text { (Hymenoptera: Braconnidae); diamond back moth and tobacco caterpillar }\end{array}$ \\
\hline Article Info & on parasitoid of Cotesia sp. (Hymenoptera: Braconidae). However, their \\
\hline $\begin{array}{l}\text { Accepted: } \\
14 \text { May } 2017 \\
\text { Available Online: } \\
10 \text { June } 2017\end{array}$ & $\begin{array}{l}\text { population were found fluctuate during the crop season. Among foliage } \\
\text { feeding pests the tobacco caterpillar incidence was higher followed by } \\
\text { diamond back moth. While, natural enemies the maximum mean population } \\
\text { was found for Coccinella septempunctata during both the years. }\end{array}$ \\
\hline
\end{tabular}

\section{Introduction}

Cruciferous vegetables have an important place among rabi crops grown in India. Cabbage, Brassica oleracea var. capitata (Linn.), is a popular vegetable that is grown in all the states of India and has appreciable nutritional and economic value. Insect pests are a serious menace in the profitable cultivation of cabbage. The important insect pests that infest cabbage crop are the tobacco caterpillar (Spodoptera litura Fab.), diamond back moth (Plutella xylostella L.), cabbage semilooper (Trichoplusia ni Hubner), painted bug (Bagrada hilaris Burmeister and Bagrada cruciferarum Kirk.), cabbage butterfly (Pieris brassicae L.), flea beetle (Phyllotreta cruciferae Goeze), aphids (Lipaphis erysimi Kalt. and Brevicoryne brassicae L.), Cabbage leaf webber (Crocidolomia bionotalis Zell) and the mustard saw fly (Athalia lugens proxima Klug.) (Ahuja, et al., 2012; Alam, 1992). Therefore, the present study was undertaken in the cabbage growing area to the 
insect biodiversity associated with this system. The major natural enemies are coccinellids, Coccinella septempunctata (Fab.), Cheilomenes sexmaculatus (Fab.) (Coleoptera: Coccinellidae); common green lacewing, Chrysoperla sp. (Neuroptera: Chrysopidae); aphid parasitoid, Diaeretiella sp. (Hymenoptera: Braconnidae); diamond back moth and tobacco caterpillar on parasitoid of Cotesia sp. (Hymenoptera: Braconidae), C. septempunctata.

\section{Materials and Methods}

The experiment was carried out during rabi, 2012-13 and 2013-14 in cabbage variety Golden Acre. The experimental site is situated at Horticulture Farm, Rajasthan College of Agriculture, Maharana Pratap University of Agriculture and Technology, Udaipur. Weekly meteorological data were obtained from the Meteorology Unit at the Instructional farm of the College. All the normal agronomic practices were followed for raising the crop. The experiment was replicated thrice in a randomized block design. The observation on tobacco caterpillar, Spodoptera litura (Fab.), diamond back moth, Plutella xylostella (Linn.), cabbage semilooper, Thysanoplusia orichalcea (Fab.) and flea beetle, Phyllotreta chotanica (Duviv), coccinellids, Coccinella septempunctata (Fab.), Cheilomenes sexmaculatus (Fab.); common green lacewing, Chrysoperla sp., direct visual counting method was used and population was recorded on ten plants selected randomly from each replicate at weekly intervals. The estimation of aphid population was based on the numerical count method as described. The population was counted only on three leaves. For recording the aphid population marked leaves were grasped at the petiole by thumb and fore finger and twisted until entire underside of the leaves were clearly visible. The aphid population was counted weekly with the help of magnifying lens. To record the larval parasitization of $P$. xylostella and $S$. litura known numbers of larvae were collected from cabbage field at weekly intervals brought to the laboratory and reared in glass jars (500ml capacity) separatly on fresh cabbage leaves. The jars were covered with a muslin cloth secured with rubber bands and placed in the laboratory at ambient conditons of temperature and humidity. The larvae were observed upto the emergence of parasitoids and the numbers of parasitization larvae were counted. The emerged parasitoids from larvae were identified. The seasonal mean population counts of different insect pests and their natural enemies.

\section{Results and Discussion}

\section{Impact of Cotesia sp. on $S$. litura in cabbage}

The population of Cotesia sp. touched its peak (0.70 adult/plant) during 5th SMW when the $S$. litura population also was at its peak (5.40 larvae/plant) and then gradually declined. Cotesia sp. population exhibited a significant and positive correlation $(\mathrm{r}=0.907)$ with the $S$. litura population during rabi 201213 (Table 1). Similarly, during rabi 2013-14 Cotesia sp. population gradually increased and touched its peak ( 0.70 adult/plant) during 5th SMW when the $S$. litura population was also (5.80 adult/plant) (Table 2). The parasitoid of $S$. lituar, Cotesia sp. was recorded in field from field collected larvae of $S$. lituar and the parasitisation ranged from 4.54 to 12.96 per cent during 2012-13 and 5.82 to 12.07 per cent during 2013-14. The influence of the parasitoid Cotesia sp on S. litura was moderate and stalle with 12.96 and 12.07 per cent parasitisation during rabi 201213 and 2013-14, respectively. Cotesia sp. population exhibited a significant and positive correlation ( $r=0.907$ and $r=0.914)$ with $S$. litura, respectively, during rabi 2012-13 and 
2013-14. Joshi et al., (1979) reported that the Cotesia sp. was larval parasitoid of S. litura on cabbage and cauliflower. Krishnamoorthy and Mani (1985) reported that the larval parasitoid 14.50 per cent by Cotesia marginivetris of S. litura. Ahuja et al., (2012) reported that the larval parasitoid by Cotesia glomeratus predating neonate larvae of $S$. litura.

Impact of natural enemies on diamond back moth by Cotesia sp.

The diamond back moth parasitoid, Cotesia sp. was recorded from field collected diamond back moth larvae maintained in the laboratory during the 52nd SMW. The maximum number of parasitized larvae $(\%)$ was noted during 5th and 6th SMW.

\section{Larval parasitisation of diamond back} moth by Cotesia sp.

In 2012-13, parasitoid activity of diamond back moth was first noted in 52nd SMW with 5.00 per cent larval parasitisation that increased steadily upto 5th SMW (12.96\%) (Table 3). Similarly, in 2013-14, parasitiod activity of diamond back moth was first noted in 52nd (9.52\%) increasing upto 5th SMW (Table 4). Natural enemies of $P$. xylostella were reported by earlier workers like Kitauchi and Nogami (1984), Alam (1992), Noda et al., (1996), Talekar and Hu (1996) and Wang et al., (1998) from different areas. Cock (1985) reported 89 to 100 per cent parasitisation of diamond back moth. Liu et al., (2000) also reported that Cotesia sp. was the key factor in control of natural population of diamond back moth.

Impact of natural enemies on aphid in cabbage

\section{Coccinella septempunctata (Fab.)}

The population touched its peak $(2.10$ adult/plant) during 6th SMW when the aphid population also was at its peak (40.80 adult/plant) and then gradually declined. The mean temperature and relative humidity at peak period of activity were, $15.95^{\circ} \mathrm{C}$ and 53.05 per cent, respectively. $C$. septempunctata population exhibited a significant and positive correlation $(r=0.977)$ with the aphid population during rabi 2012-13 (Table 5). Similarly, during rabi 2013-14 C. septempunctata population gradually increased and touched its peak (2.10 adult/plant) during 7th SMW when the aphid population was also highest (42.40 adult/plant) (Table 6). The mean temperature and relative humidity at peak period of activity were, $15.45^{\circ} \mathrm{C}$ and 60.55 per cent, respectively. C. septempunctata population exhibited a significant and positive correlation $(\mathrm{r}=0.823)$ with aphid population during rabi 2013-14. The present findings are in agreement with that of Kumawat (2004) who reported that $C$. septempunctata was active in the second week of December and thereafter reached to peak in second week of February on the crop transplanted in October. Vekaria and Patel (2005), Mandal and Patnaik (2008), Arshad and Rana (2012) and Patra et al., (2013) reported the highest population of $C$. septempunctata in the third week of February. Kulkarni and Patel (2001) assessed that $C$. septempunctata was active in the last week of January and afterward reached to peak level, partially supporting findings.

C. septempunctata had negative correlation $(\mathrm{r}=-0.071$ and $\mathrm{r}=-0.019)$ with mean temperature and relative humidity $(\mathrm{r}=0.067$ and $r=0.126$ ) during rabi 2012-13 and 201314. The present findings are in agreement with the study of Kumawat (2004) who reported that $C$. septempunctata exhibited a negative correlation with temperature and positive correlation with relative humidity. Kumar et al., (2009) reported that $C$. septempunctata exhibited a negative correlation with maximum and minimum 
temperature and minimum relative humidity but significant positive correlation with maximum relative humidity.

\section{Cheilomenes sexmaculata (Fab.)}

C. sexmaculata was observed from first week of January (1st SMW) to last week of February (9th SMW) during rabi 2012-13 (Table 5). The population increased upto 1.80 adult/plant during 6th SMW when the aphid population also peaked (40.80 adult/plant). The mean temperature and relative humidity at peak period of activity were, $15.95^{\circ} \mathrm{C}$ and 53.05 per cent, respectively. C. sexmaculata population exhibited a significant and positive correlation $(r=0.978)$ with aphid. Similarly, C. sexmaculata was observed from first week of January (1st SMW) to last week of February (9th SMW) during rabi 2013-14 (Table 6). The population gradually increased reaching 2.20 adult/plant during 7th SMW when aphid population was also at its peak (42.40 adult/plant). The mean temperature and relative humidity at peak period of activity were, $15.45^{\circ} \mathrm{C}$ and 60.55 per cent, respectively.

C. sexmaculata population exhibited an significant and positive correlation with $(\mathrm{r}=$ 0.770) aphids during rabi 2013-14. The present results are in agreement with that of Mandal and Patnaik (2008) who reported the peak population during last week of January and February and Patra et al., (2013) who reported the peak population of $M$. sexmaculata during third week of February. Partially supporting the present findings Singh et al., (2006) assessed that $M$. sexmaculata was active in the 8th SMW and afterward reached to peak level, whereas, Gour (2001) reported that M. sexmaculata reached to its peak in the fourth week of January.

C. sexmaculata population exhibited a negative correlation $(r=-0.102$ and $r=-0.041)$ with mean temperature and positive correlation with mean relative humidity $(\mathrm{r}=$ 0.053 and $r=0.128$ ) during rabi 2012-13 and 2013-14. Earlier, Kumawat (2004) reported negative correlation with temperature and relative humidity on cabbage transplanted in October. Gour (2001) and Mishra (2003) reported negative correlation with temperature and positive correlation with relative humidity on mustard, partially supporting the present findings.

\section{Mummified aphid}

The numbers of mummified aphids were maximum (10.60 mummified aphids /plant) in the 8th SMW during rabi 2012-13 (Table 5), while (10.80 mummified aphids /plant) in the 8th SMW during rabi 2013-14 (Table 6).

The mummified aphids exhibited a significant and positive correlation $(\mathrm{r}=0.754$ and $\mathrm{r}=$ 0.828 ) with aphid during both years indicating a density dependent effect. The present findings are supported by Kulkarni and Patel (2001) who reported the appearance of the mummified aphid in the first week of February that touched peak in the third week of February.

Vekaria (1998) also reported the maximum activity of this parasite in the fourth week of February. Hugar et al., (2008) reported the maximum numbers of mummified aphids in the last week of February. Kumawat (2004) reported that the parasitoids were active in the last week of December and reached to a peak in second week of February on cabbage transplanted in October.

Kumar (1989) found mummified aphid as a major parasite on mustard aphid, L. erysimi, in second week of December which slowly increased to its peak during second week of February. 
Table.1 Seasonal incidence and population dynamics of tobacco caterpillar, Spodoptera litura (Fab.) with their natural enemy and abiotic factors in cabbage ecosystem during rabi 2012-13

\begin{tabular}{|c|c|c|c|c|c|c|c|c|c|c|}
\hline \multirow[t]{3}{*}{ SMW } & \multirow{3}{*}{$\begin{array}{c}\text { Dates of } \\
\text { Observation }\end{array}$} & \multicolumn{6}{|c|}{ Abiotic factors } & \multicolumn{2}{|c|}{ Mean population/plant } & \multirow{3}{*}{$\begin{array}{c}\text { Mean } \\
\text { parasitoid } \\
\text { population } \\
(\%)\end{array}$} \\
\hline & & \multicolumn{3}{|c|}{ Temperature $\left({ }^{\circ} \mathrm{C}\right)$} & \multicolumn{3}{|c|}{ Relative Humidity (\%) } & \multirow{2}{*}{$\begin{array}{c}\text { Tobacco } \\
\text { caterpillar }\end{array}$} & \multirow[t]{2}{*}{ Cottesia sp. } & \\
\hline & & Max & Min & Mean & $\operatorname{Max}$ & Min & Mean & & & \\
\hline 50 & $14 / 12 / 2012$ & 27.90 & 10.30 & 19.10 & 81.10 & 27.10 & 54.10 & 1.10 & 0.00 & 0.00 \\
\hline 51 & $21 / 12 / 2012$ & 25.90 & 7.70 & 16.80 & 82.00 & 25.40 & 53.70 & 1.80 & 0.00 & 0.00 \\
\hline 52 & $28 / 12 / 2012$ & 25.30 & 7.00 & 16.15 & 76.10 & 27.10 & 51.60 & 2.20 & 0.10 & 4.54 \\
\hline 1 & $04 / 12 / 2013$ & 23.00 & 4.40 & 13.70 & 85.60 & 30.70 & 58.15 & 2.80 & 0.20 & 7.14 \\
\hline 2 & $11 / 01 / 2013$ & 25.10 & 6.40 & 15.70 & 75.30 & 21.10 & 48.20 & 3.10 & 0.30 & 9.68 \\
\hline 3 & $18 / 01 / 2013$ & 23.90 & 7.50 & 15.70 & 80.30 & 28.10 & 54.20 & 4.50 & 0.40 & 8.89 \\
\hline 4 & $25 / 01 / 2013$ & 22.70 & 4.00 & 13.35 & 74.90 & 19.60 & 47.25 & 4.60 & 0.40 & 8.70 \\
\hline 5 & $01 / 022013$ & 25.90 & 10.00 & 17.95 & 76.30 & 26.90 & 51.60 & 5.40 & 0.70 & 12.96 \\
\hline 6 & $08 / 02 / 2013$ & 24.30 & 7.60 & 15.95 & 78.00 & 28.10 & 53.05 & 4.80 & 0.50 & 10.42 \\
\hline 7 & $15 / 02 / 2013$ & 26.30 & 11.70 & 19.00 & 83.30 & 34.30 & 58.80 & 4.50 & 0.50 & 11.11 \\
\hline 8 & $22 / 02 / 2013$ & 26.80 & 10.60 & 18.70 & 82.00 & 31.00 & 56.50 & 3.60 & 0.40 & 11.11 \\
\hline 9 & $29 / 02 / 2013$ & 29.00 & 8.70 & 18.85 & 65.90 & 18.40 & 42.15 & 2.40 & 0.30 & 12.50 \\
\hline \multicolumn{2}{|c|}{ Seasonal Mean } & 25.51 & 7.99 & 16.74 & 78.40 & 26.48 & 52.44 & $3.40(11.33)$ & 0.38 (1.27) & 9.75 \\
\hline \multicolumn{8}{|c|}{ Relative Density (\%) } & 29.37 & - & - \\
\hline \multicolumn{11}{|c|}{ Correlation coefficient between population of insect pests, abiotic and biotic factors } \\
\hline \multicolumn{8}{|c|}{ Mean temperature $\left({ }^{0} \mathrm{C}\right)$} & -0.192 & 0.048 & - \\
\hline \multicolumn{8}{|c|}{ Mean relative humidity $(\%)$} & 0.084 & -0.014 & - \\
\hline \multicolumn{8}{|c|}{ Correlation coefficient between population of $S$. litura and their natural enemy } & - & $0.907 *$ & - \\
\hline
\end{tabular}


Table.2 Seasonal incidence and population dynamics of tobacco caterpillar, Spodoptera litura (Fab.) with their natural enemy and abiotic factors in cabbage ecosystem during rabi 2013-14

\begin{tabular}{|c|c|c|c|c|c|c|c|c|c|c|}
\hline \multirow[t]{3}{*}{ SMW } & \multirow{3}{*}{$\begin{array}{c}\text { Dates of } \\
\text { Observation }\end{array}$} & \multicolumn{6}{|c|}{ Abiotic factors } & \multicolumn{2}{|c|}{ Mean population/plant } & \multirow{3}{*}{$\begin{array}{c}\text { Mean } \\
\text { parasitoid } \\
\text { population } \\
(\%)\end{array}$} \\
\hline & & \multicolumn{3}{|c|}{ Temperature $\left({ }^{\circ} \mathrm{C}\right)$} & \multicolumn{3}{|c|}{ Relative Humidity (\%) } & \multirow{2}{*}{$\begin{array}{c}\text { Tobacco } \\
\text { caterpillar }\end{array}$} & \multirow{2}{*}{ Cotesia sp. } & \\
\hline & & $\operatorname{Max}$ & Min & Mean & Max & Min & Mean & & & \\
\hline 50 & $14 / 12 / 2013$ & 27.40 & 8.20 & 17.80 & 81.70 & 25.80 & 53.75 & 1.20 & 0.00 & 0.00 \\
\hline 51 & $21 / 12 / 2013$ & 24.70 & 7.00 & 15.85 & 85.60 & 32.80 & 59.20 & 2.10 & 0.00 & 0.00 \\
\hline 52 & $28 / 12 / 2013$ & 22.20 & 7.70 & 14.95 & 84.17 & 42.20 & 63.18 & 2.60 & 0.20 & 7.69 \\
\hline 1 & $04 / 01 / 2014$ & 22.20 & 7.50 & 14.85 & 77.40 & 44.10 & 60.75 & 3.40 & 0.20 & 5.88 \\
\hline 2 & $11 / 01 / 2014$ & 21.50 & 6.40 & 13.95 & 84.30 & 43.40 & 63.85 & 3.70 & 0.30 & 8.11 \\
\hline 3 & $18 / 01 / 2014$ & 22.20 & 6.60 & 14.40 & 88.00 & 47.10 & 67.55 & 4.20 & 0.40 & 9.52 \\
\hline 4 & $25 / 01 / 2014$ & 21.40 & 9.70 & 15.55 & 89.70 & 49.10 & 69.40 & 4.80 & 0.40 & 8.33 \\
\hline 5 & $01 / 02 / 2014$ & 26.30 & 8.50 & 17.40 & 87.70 & 29.70 & 58.70 & 5.10 & 0.50 & 9.80 \\
\hline 6 & $08 / 02 / 2014$ & 26.80 & 9.80 & 18.30 & 76.80 & 23.40 & 50.10 & 5.80 & 0.70 & 12.07 \\
\hline 7 & $15 / 02 / 2014$ & 23.00 & 7.90 & 15.45 & 85.10 & 36.00 & 60.55 & 5.20 & 0.40 & 7.70 \\
\hline 8 & $22 / 02 / 2014$ & 25.50 & 11.00 & 18.25 & 85.60 & 40.00 & 62.80 & 3.50 & 0.30 & 8.57 \\
\hline 9 & $29 / 02 / 2014$ & 26.80 & 8.80 & 17.80 & 86.60 & 31.30 & 58.95 & 2.50 & 0.20 & 8.00 \\
\hline \multicolumn{2}{|c|}{ Seasonal Mean } & 24.17 & 8.26 & 16.21 & 84.39 & 37.08 & 60.73 & $3.67(12.23)$ & $0.36(1.20)$ & 9.81 \\
\hline \multicolumn{8}{|c|}{ Relative Density (\%) } & 32.50 & - & - \\
\hline \multicolumn{11}{|c|}{ Correlation coefficient between population of insect pests, abiotic and biotic factors } \\
\hline \multicolumn{8}{|c|}{ Mean temperature $\left({ }^{0} \mathrm{C}\right)$} & -0.026 & 0.138 & - \\
\hline \multicolumn{8}{|c|}{ Mean relative humidity $(\%)$} & 0.078 & -0.055 & - \\
\hline \multicolumn{8}{|c|}{$\begin{array}{l}\text { Correlation coefficient between population of } S \text {. litura and their natural } \\
\text { enemy }\end{array}$} & - & $0.914 *$ & - \\
\hline
\end{tabular}

SMW- Standard meteorological week, Figures in parentheses are mean density values $(\%),{ }^{*}$ denotes ${ }^{\mathrm{R}}$-value significant at $\mathrm{p}=0.05$ 
Table.3 Seasonal incidence and population dynamics of diamond back moth, Plutella xylostella (linn.) With their natural enemy and abiotic factors in cabbage ecosystem during rabi 2012-13

\begin{tabular}{|c|c|c|c|c|c|c|c|c|c|c|}
\hline \multirow[t]{3}{*}{ SMW } & \multirow{3}{*}{$\begin{array}{c}\text { Dates of } \\
\text { Observation }\end{array}$} & \multicolumn{6}{|c|}{ Abiotic factors } & \multicolumn{2}{|c|}{ Mean population/plant } & \multirow{3}{*}{$\begin{array}{c}\text { Mean } \\
\text { parasitoid } \\
\text { population } \\
(\%)\end{array}$} \\
\hline & & \multicolumn{3}{|c|}{ Temperature $\left({ }^{\circ} \mathrm{C}\right)$} & \multicolumn{3}{|c|}{ Relative Humidity (\%) } & \multirow{2}{*}{$\begin{array}{c}\text { Diamond } \\
\text { back moth }\end{array}$} & \multirow[t]{2}{*}{ Cotesia sp. } & \\
\hline & & Max & Min & Mean & Max & Min & Mean & & & \\
\hline 50 & $14 / 12 / 2012$ & 27.90 & 10.30 & 19.10 & 81.10 & 27.10 & 54.10 & 1.20 & 0.00 & 0.00 \\
\hline 51 & $21 / 12 / 2012$ & 25.90 & 7.70 & 16.80 & 82.00 & 25.40 & 53.70 & 1.70 & 0.00 & 0.00 \\
\hline 52 & $28 / 12 / 2012$ & 25.30 & 7.00 & 16.15 & 76.10 & 27.10 & 51.60 & 2.00 & 0.10 & 5.00 \\
\hline 1 & $04 / 12 / 2013$ & 23.00 & 4.40 & 13.70 & 85.60 & 30.70 & 58.15 & 2.20 & 0.20 & 9.09 \\
\hline 2 & $11 / 01 / 2013$ & 25.10 & 6.40 & 15.70 & 75.30 & 21.10 & 48.20 & 3.50 & 0.30 & 8.57 \\
\hline 3 & $18 / 01 / 2013$ & 23.90 & 7.50 & 15.70 & 80.30 & 28.10 & 54.20 & 3.60 & 0.40 & 11.11 \\
\hline 4 & $25 / 01 / 2013$ & 22.70 & 4.00 & 13.35 & 74.90 & 19.60 & 47.25 & 3.70 & 0.40 & 10.81 \\
\hline 5 & $01 / 022013$ & 25.90 & 10.00 & 17.95 & 76.30 & 26.90 & 51.60 & 5.40 & 0.70 & 12.96 \\
\hline 6 & $08 / 02 / 2013$ & 24.30 & 7.60 & 15.95 & 78.00 & 28.10 & 53.05 & 4.50 & 0.50 & 11.11 \\
\hline 7 & $15 / 02 / 2013$ & 26.30 & 11.70 & 19.00 & 83.30 & 34.30 & 58.80 & 4.40 & 0.50 & 11.36 \\
\hline 8 & $22 / 02 / 2013$ & 26.80 & 10.60 & 18.70 & 82.00 & 31.00 & 56.50 & 4.30 & 0.40 & 9.30 \\
\hline 9 & $29 / 02 / 2013$ & 29.00 & 8.70 & 18.85 & 65.90 & 18.40 & 42.15 & 4.10 & 0.30 & 7.72 \\
\hline \multicolumn{2}{|c|}{ Seasonal Mean } & 25.51 & 7.99 & 16.74 & 78.40 & 26.48 & 52.44 & $3.38(11.27)$ & $0.38(1.27)$ & 10.47 \\
\hline \multicolumn{8}{|c|}{ Relative Density (\%) } & 29.33 & - & \\
\hline \multicolumn{11}{|c|}{ Correlation coefficient between population of insect pests, abiotic and biotic factors } \\
\hline \multicolumn{8}{|c|}{ Mean temperature $\left({ }^{0} \mathrm{C}\right)$} & 0.170 & 0.048 & - \\
\hline \multicolumn{8}{|c|}{ Mean relative humidity $(\%)$} & -0.165 & -0.014 & - \\
\hline \multicolumn{8}{|c|}{$\begin{array}{l}\text { Correlation coefficient between population of diamond back moth and their } \\
\text { natural enemy }\end{array}$} & & $0.930^{*}$ & - \\
\hline
\end{tabular}

SMW- Standard meteorological week, Figures in parentheses are mean density values (\%), *r-value significant at $\mathrm{p}=0.05$ 
Table.4 Seasonal incidence and population dynamics of diamond back moth, Plutella xylostella (linn.) with their natural enemy and abiotic factors in cabbage ecosystem during rabi 2013-14

\begin{tabular}{|c|c|c|c|c|c|c|c|c|c|c|}
\hline \multirow{3}{*}{ SMW } & \multirow{3}{*}{$\begin{array}{l}\text { Dates of } \\
\text { Observation }\end{array}$} & \multicolumn{6}{|c|}{ Abiotic factors } & \multicolumn{2}{|c|}{ Mean population/plant } & \multirow{3}{*}{$\begin{array}{c}\text { Mean } \\
\text { parasitoid } \\
\text { population } \\
(\%)\end{array}$} \\
\hline & & \multicolumn{3}{|c|}{ Temperature $\left({ }^{\mathbf{0}} \mathrm{C}\right)$} & \multicolumn{3}{|c|}{ Relative Humidity (\%) } & \multirow{2}{*}{$\begin{array}{c}\text { Diamond } \\
\text { back moth }\end{array}$} & \multirow{2}{*}{ Cotesia sp. } & \\
\hline & & Max & Min & Mean & $\operatorname{Max}$ & Min & Mean & & & \\
\hline 50 & $14 / 12 / 2013$ & 27.40 & 8.20 & 17.80 & 81.70 & 25.80 & 53.75 & 1.30 & 0.00 & 0.00 \\
\hline 51 & $21 / 12 / 2013$ & 24.70 & 7.00 & 15.85 & 85.60 & 32.80 & 59.20 & 1.50 & 0.00 & 0.00 \\
\hline 52 & $28 / 12 / 2013$ & 22.20 & 7.70 & 14.95 & 84.17 & 42.20 & 63.18 & 2.10 & 0.20 & 9.52 \\
\hline 1 & $04 / 01 / 2014$ & 22.20 & 7.50 & 14.85 & 77.40 & 44.10 & 60.75 & 2.50 & 0.20 & 8.00 \\
\hline 2 & $11 / 01 / 2014$ & 21.50 & 6.40 & 13.95 & 84.30 & 43.40 & 63.85 & 2.70 & 0.30 & 11.11 \\
\hline 3 & $18 / 01 / 2014$ & 22.20 & 6.60 & 14.40 & 88.00 & 47.10 & 67.55 & 3.30 & 0.40 & 12.12 \\
\hline 4 & $25 / 01 / 2014$ & 21.40 & 9.70 & 15.55 & 89.70 & 49.10 & 69.40 & 3.50 & 0.40 & 11.43 \\
\hline 5 & $01 / 02 / 2014$ & 26.30 & 8.50 & 17.40 & 87.70 & 29.70 & 58.70 & 4.50 & 0.50 & 11.11 \\
\hline 6 & $08 / 02 / 2014$ & 26.80 & 9.80 & 18.30 & 76.80 & 23.40 & 50.10 & 5.20 & 0.70 & 13.46 \\
\hline 7 & $15 / 02 / 2014$ & 23.00 & 7.90 & 15.45 & 85.10 & 36.00 & 60.55 & 4.00 & 0.40 & 10.00 \\
\hline 8 & $22 / 02 / 2014$ & 25.50 & 11.00 & 18.25 & 85.60 & 40.00 & 62.80 & 3.70 & 0.30 & 8.11 \\
\hline 9 & $29 / 02 / 2014$ & 26.80 & 8.80 & 17.80 & 86.60 & 31.30 & 58.95 & 2.90 & 0.20 & 6.90 \\
\hline \multicolumn{2}{|c|}{ Seasonal Mean } & 24.17 & 8.26 & 16.21 & 84.39 & 37.08 & 60.73 & $3.10(10.33)$ & $0.36(1.20)$ & 10.18 \\
\hline \multicolumn{8}{|c|}{ Relative Density (\%) } & 27.41 & & \\
\hline \multicolumn{11}{|c|}{ Correlation coefficient between population of insect pests, abiotic and biotic factors } \\
\hline \multicolumn{8}{|c|}{ Mean temperature $\left({ }^{0} \mathrm{C}\right)$} & 0.309 & 0.138 & - \\
\hline \multicolumn{8}{|c|}{ Mean relative humidity $(\%)$} & -0.110 & -0.055 & - \\
\hline \multicolumn{8}{|c|}{$\begin{array}{l}\text { Correlation coefficient between population of Diamond back moth and their } \\
\text { natural enemy }\end{array}$} & - & $0.923 *$ & - \\
\hline
\end{tabular}

SMW- Standard meteorological week, Figures in parentheses are mean density values (\%), *r-value significant at $\mathrm{p}=0.05$ 
Table.5 Seasonal incidence and population dynamics of aphid, Lipaphis erysimi (Kalt.) with their natural enemies and Abiotic factors in cabbage ecosystem during rabi 2012-13

\begin{tabular}{|c|c|c|c|c|c|c|c|c|c|c|c|c|}
\hline \multirow{3}{*}{ SMW } & \multirow{3}{*}{$\begin{array}{c}\text { Dates of } \\
\text { Observation }\end{array}$} & \multicolumn{6}{|c|}{ Abiotic factors } & \multirow{3}{*}{ Aphid } & \multicolumn{3}{|c|}{ Mean Predator Population/plant } & \multirow{3}{*}{$\begin{array}{c}\begin{array}{c}\text { Mean Parasitoid } \\
\text { Population/Plant }\end{array} \\
\begin{array}{c}\text { Mummified } \\
\text { aphids/plant }\end{array}\end{array}$} \\
\hline & & \multicolumn{3}{|c|}{ Temperature $\left({ }^{0} \mathrm{C}\right)$} & \multicolumn{3}{|c|}{$\begin{array}{c}\text { Relative Humidity } \\
(\%)\end{array}$} & & \multirow{2}{*}{$\begin{array}{c}\text { Coccinella } \\
\text { septempunctat } \\
a\end{array}$} & \multirow[t]{2}{*}{$\begin{array}{l}\text { Cheilomenes } \\
\text { sexmaculata }\end{array}$} & \multirow[t]{2}{*}{$\begin{array}{c}\text { Chrysoperla } \\
\text { sp. }\end{array}$} & \\
\hline & & Max & Min & Mean & Max & Min & Mean & & & & & \\
\hline 50 & $14 / 12 / 2013$ & 27.90 & 10.30 & 19.10 & 81.10 & 27.10 & 54.10 & 0.00 & 0.00 & 0.00 & 0.00 & 0.00 \\
\hline 51 & $21 / 12 / 2013$ & 25.90 & 7.70 & 16.80 & 82.00 & 25.40 & 53.70 & 5.20 & 0.00 & 0.00 & 0.00 & 0.00 \\
\hline 52 & $28 / 12 / 2013$ & 25.30 & 7.00 & 16.15 & 76.10 & 27.10 & 51.60 & 8.40 & 0.20 & 0.00 & 0.00 & 0.00 \\
\hline 1 & $04 / 01 / 2014$ & 23.00 & 4.40 & 13.70 & 85.60 & 30.70 & 58.15 & 15.30 & 0.40 & 0.30 & 0.00 & 0.00 \\
\hline 2 & $11 / 01 / 2014$ & 25.10 & 6.40 & 15.70 & 75.30 & 21.10 & 48.20 & 16.60 & 0.90 & 0.70 & 0.10 & 4.90 \\
\hline 3 & $18 / 01 / 2014$ & 23.90 & 7.50 & 15.70 & 80.30 & 28.10 & 54.20 & 20.80 & 1.20 & 0.90 & 0.30 & 5.50 \\
\hline 4 & $25 / 01 / 2014$ & 22.70 & 4.00 & 13.35 & 74.90 & 19.60 & 47.25 & 29.70 & 1.40 & 1.20 & 0.40 & 6.20 \\
\hline 5 & $01 / 02 / 2014$ & 25.90 & 10.00 & 17.95 & 76.30 & 26.90 & 51.60 & 34.10 & 1.70 & 1.50 & 0.60 & 7.50 \\
\hline 6 & $08 / 02 / 2014$ & 24.30 & 7.60 & 15.95 & 78.00 & 28.10 & 53.05 & 40.80 & 2.10 & 1.80 & 0.80 & 7.80 \\
\hline 7 & $15 / 02 / 2014$ & 26.30 & 11.70 & 19.00 & 83.30 & 34.30 & 58.80 & 28.20 & 1.60 & 1.20 & 0.50 & 9.80 \\
\hline 8 & $22 / 02 / 2014$ & 26.80 & 10.60 & 18.70 & 82.00 & 31.00 & 56.50 & 22.60 & 1.10 & 0.80 & 0.30 & 10.60 \\
\hline 9 & $29 / 02 / 2014$ & 29.00 & 8.70 & 18.85 & 65.90 & 18.40 & 42.15 & 12.40 & 0.50 & 0.30 & 0.20 & 6.70 \\
\hline \multicolumn{2}{|c|}{ Seasonal Mean } & 25.51 & 7.99 & 16.74 & 78.40 & 26.48 & 52.44 & 21.28 & $1.11(3.70)$ & $0.97(3.23)$ & $0.40(1.33)$ & $7.37(24.57)$ \\
\hline \multicolumn{8}{|c|}{ Relative Density (\%) } & - & 56.06 & 43.94 & - & - \\
\hline \multicolumn{13}{|c|}{ Correlation coefficient between population of insect pests and abiotic factors } \\
\hline \multicolumn{8}{|c|}{ Mean temperature $\left({ }^{0} \mathrm{C}\right)$} & -0.181 & -0.071 & -0.102 & 0.075 & 0.32 \\
\hline \multicolumn{8}{|c|}{ Mean relative humidity $(\%)$} & 0.060 & 0.0671 & 0.053 & 0.025 & -0.04 \\
\hline \multicolumn{9}{|c|}{ Correlation coefficient values ( $r$ ) values for aphids and their natural enemies } & $0.977 * *$ & $0.978 * *$ & $0.952 * *$ & $0.754 *$ \\
\hline
\end{tabular}

SMW-Standard meteorological week, Figures in parentheses are mean density values (\%), ${ }^{\mathrm{r}}$-value significant at $\mathrm{p}=0.05$ 
Table.6 Seasonal incidence and population dynamics of aphid, Lipaphis erysimi (Kalt.) with their natural enemies and Abiotic factors in cabbage ecosystem during rabi 2013-14

\begin{tabular}{|c|c|c|c|c|c|c|c|c|c|c|c|c|}
\hline \multirow[t]{3}{*}{ SMW } & \multirow{3}{*}{$\begin{array}{c}\text { Dates of } \\
\text { Observation }\end{array}$} & \multicolumn{6}{|c|}{ Abiotic factors } & \multirow[t]{3}{*}{ Aphid } & \multicolumn{3}{|c|}{ Mean Predator Population/plant } & \multirow{3}{*}{$\begin{array}{c}\text { Mean Parasitoid } \\
\text { Population/Plant } \\
\begin{array}{c}\text { Mummified } \\
\text { aphids/plant }\end{array} \\
\end{array}$} \\
\hline & & \multicolumn{3}{|c|}{ Temperature $\left({ }^{\circ} \mathrm{C}\right)$} & \multicolumn{3}{|c|}{ Relative Humidity (\%) } & & \multirow{2}{*}{$\begin{array}{c}\text { Coccinella } \\
\text { septempunctata }\end{array}$} & \multirow{2}{*}{$\begin{array}{l}\text { Cheilomenes } \\
\text { sexmaculata }\end{array}$} & \multirow{2}{*}{$\begin{array}{c}\text { Chrysoperla } \\
\text { sp. }\end{array}$} & \\
\hline & & Max & Min & Mean & Max & Min & Mean & & & & & \\
\hline 50 & $14 / 12 / 2013$ & 27.40 & 8.20 & 17.80 & 81.70 & 25.80 & 53.75 & 0.00 & 0.00 & 0.00 & 0.00 & 0.00 \\
\hline 51 & $21 / 12 / 2013$ & 24.70 & 7.00 & 15.85 & 85.60 & 32.80 & 59.20 & 7.40 & 0.00 & 0.00 & 0.00 & 0.00 \\
\hline 52 & $28 / 12 / 2013$ & 22.20 & 7.70 & 14.95 & 84.17 & 42.20 & 63.18 & 10.40 & 0.30 & 0.00 & 0.00 & 0.00 \\
\hline 1 & $04 / 01 / 2014$ & 22.20 & 7.50 & 14.85 & 77.40 & 44.10 & 60.75 & 11.40 & 0.80 & 0.40 & 0.00 & 0.00 \\
\hline 2 & $11 / 01 / 2014$ & 21.50 & 6.40 & 13.95 & 84.30 & 43.40 & 63.85 & 18.30 & 1.20 & 1.20 & 0.30 & 5.20 \\
\hline 3 & $18 / 01 / 2014$ & 22.20 & 6.60 & 14.40 & 88.00 & 47.10 & 67.55 & 25.50 & 1.30 & 1.20 & 0.40 & 5.40 \\
\hline 4 & $25 / 01 / 2014$ & 21.40 & 9.70 & 15.55 & 89.70 & 49.10 & 69.40 & 28.60 & 1.50 & 1.30 & 0.60 & 5.50 \\
\hline 5 & $01 / 02 / 2014$ & 26.30 & 8.50 & 17.40 & 87.70 & 29.70 & 58.70 & 24.90 & 1.60 & 1.40 & 0.70 & 8.50 \\
\hline 6 & $08 / 02 / 2014$ & 26.80 & 9.80 & 18.30 & 76.80 & 23.40 & 50.10 & 22.50 & 1.80 & 1.50 & 0.50 & 8.90 \\
\hline 7 & $15 / 02 / 2014$ & 23.00 & 7.90 & 15.45 & 85.10 & 36.00 & 60.55 & 42.40 & 2.10 & 2.20 & 0.40 & 9.20 \\
\hline 8 & $22 / 02 / 2014$ & 25.50 & 11.00 & 18.25 & 85.60 & 40.00 & 62.80 & 23.90 & 1.20 & 1.00 & 0.30 & 10.80 \\
\hline 9 & $29 / 02 / 2014$ & 26.80 & 8.80 & 17.80 & 86.60 & 31.30 & 58.95 & 15.10 & 0.50 & 0.40 & 0.20 & 6.90 \\
\hline \multicolumn{2}{|c|}{ Seasonal Mean } & 24.17 & 8.26 & 16.21 & 84.39 & 37.08 & 60.73 & $\begin{array}{c}20.94 \\
(69.80) \\
\end{array}$ & $1.23(4.10)^{*}$ & $1.18(3.93)$ & $0.42(1.40)$ & 7.79 \\
\hline \multicolumn{8}{|c|}{ Relative Density (\%) } & - & 53.71 & 46.29 & - & \\
\hline \multicolumn{13}{|c|}{ Correlation coefficient between population of insect pests and abiotic factors } \\
\hline \multicolumn{8}{|c|}{ Mean temperature $\left({ }^{0} \mathrm{C}\right)$} & -0.107 & -0.019 & -0.041 & 0.160 & 0.41 \\
\hline \multicolumn{8}{|c|}{ Mean relative humidity $(\%)$} & $\mathbf{0 . 3 3 0}$ & 0.126 & 0.128 & 0.159 & -0.01 \\
\hline \multicolumn{9}{|c|}{ Correlation coefficient values ( $r$ ) values for aphids and their natural enemies } & $0.823 * *$ & $0.770 * *$ & $\mathbf{0 . 3 3 2}$ & $\mathbf{0 . 8 2 8} *$ \\
\hline
\end{tabular}

SMW-Standard meteorological week, Figures in parentheses are mean density values (\%), *r-value significant at $\mathrm{p}=0.05$ 
Kumar (2009) recorded that mummified aphid was maximum in the 3rd week of January. Singh and Rawat (1981) observed that mummified aphids were observed from second week or third week of January and reached to its peak in the fourth week of February. The parasites of mummified aphids showed the positive correlation $(r=0.320$ and $r=0.410)$ with mean temperature and had negative correlation with mean relative humidity $(\mathrm{r}=$ 0.040and $\mathrm{r}=-0.010$ ) during rabi 2012-13 and 2013-14. Kumar et al., (2009) reported that the parasitoids of mummified aphids exhibited a negative correlation with mean relative humidity.

\section{Chrysoperla sp.}

The data indicate that Chrysoperla sp. was observed from second week of January (2nd SMW) upto last week of February (9th SMW) and aphid was observed from third week of December (51st SMW) upto last week of February (9th SMW) during rabi 2012-13. The population gradually increased up to $(0.80$ adults/plant) and, whereas, aphid populationat at peak period of 40.80 aphids/plant during 6th SMW. The mean temperature and relative humidity during peak were $15.95^{\circ} \mathrm{C}$ and 53.05 per cent respectively. The Chrysoperla sp. population exhibited a significant and positive correlation ( $\mathrm{r}=0.952)$ with aphid, whereas, the Chrysoperla sp. population exhibited a nonsignificant and positive correlation $(r=0.075)$ with mean temperature and non-significant and negative correlation with mean relative humidity $(\mathrm{r}=-0.014)$ during rabi 2012-13 (Table 5). During rabi 2013-14, Chrysoperla sp. was observed from second week of January (2nd SMW) and aphid was observed from third week of December (51st SMW) to last week of February (9th SMW). The population gradually increased up to (0.70 adult/plant) and, whereas, aphid population observed 34.10 aphids/plant during 5th SMW (Table 6). The mean temperature and relative humidity at peak period of activity were, $17.40^{\circ} \mathrm{C}$ and 58.70 per cent, respectively. The Chrysoperla sp. population exhibited a non-significant and positive correlation $(\mathrm{r}=0.332)$ with aphid, whereas, the Chrysoperla sp. population exhibited a non-significant and positive correlation $(r=0.138)$ with mean temperature and non-significant and negative correlation with mean relative humidity $(\mathrm{r}=-0.055)$ during rabi 2013-14. The present results are in agreement with that of Kumawat (2004) who reported that Chrysoperla sp. appeared in the second week of January and reached to peak in the second week of February on the crop transplanted in October. Chrysoperla sp. population exhibited a positive correlation $(\mathrm{r}=$ 0.075 and $r=0.160$ ) with mean temperature and relative humidity $(\mathrm{r}=0.025$ and $\mathrm{r}=0.159)$ during rabi 2012-13 and 2013-14. The present results are in agreement with that of Kumawat (2004) who reported that Chrysoperla sp. exhibited a positive correlation with minimum temperature and evening relative humidity on the cabbage crop in October.

It was observed that the predator C. septempunctata was significantly more predating aphid in cabbage than other predators; C. sexmaculata and Chrysoperla sp. with seasonal mean density of -3.70 and 4.10 per cent during rabi 2012-13 and 2013-14, respectively. The population of Chrysoperla sp. was initiation from the second week of January and reached to its peak 0.80 and 0.70 adult/plant in the 6th and 5th SMW during rabi 2012-13 and 2013-14, respectively. Chrysoperla sp. population exhibited a positive correlation with mean temperature and relative humidity during rabi 2012-13 and 2013-14. The aphid parasitoids as mummified aphids appeared in the second week of January and were the maximum (10.60 and 10.80 adult/plant) during 5th and 6th SMW during rabi 2012-13 and 2013-14, respectively. Among aphid feeding coccinellids, $C$. septempunctata had higher mean density than $C$. sexmaculata being 56.06 and 53.71 per cent during rabi 2012-13 and 2013-14, respectively. Cotesia sp. the population touched its peak $(0.70$ and 0.70 adult/plant) during 5th SMW when the S. litura population also was at its peak (5.40 and 5.80 larvae/plant) and then gradually 
declined. Cotesia sp. population had significant and positive correlation $(\mathrm{r}=0.907$ and $\mathrm{r}=0.914)$ with the S. litura population during rabi 201213 and 20113-14. The parasitoid of S. lituar, Cotesia sp. was recorded in field from field collected larve of S. lituar and the parasitisation ranged from 4.54 to 12.96 per cent during 201213 and 5.82 to 12.07 per cent during 2013-14. The influence of the parasitoid Cotesia sp. on $\mathrm{S}$. lituar was moderate and stalle with 12.96 and 12.07 per cent parasitisation during rabi 201213 and 2013-14, respectively. Cotesia sp. population exhibited a significant and positive correlation ( $\mathrm{r}=0.907$ and $\mathrm{r}=0.914)$ with S. litura, respectively, during rabi 2012-13 and 2013-14.

\section{Acknowledgement}

The authors are thankful to the Dean, Rajasthan College of Agriculture, Maharana Pratap University of Agriculture and Technology, Udaipur, Rajasthan (India) for providing necessary facilities and permission to conduct the study.

\section{References}

Ahuja, D.B., Ahuja, Usha Rani, Srinivas, P., Singh, R.V., Malik, Meenakshi., Sharma, Pratibha and Bamawale, O.M. 2012. Development of farmer-led integrated management of major pests of cauliflower cultivated in rainy season in India. $J$. Agri. Sci., 4(2): 79-90.

Alam, M.M. 1992. The dimond back moth and its natural enemies in Jamaica and other Caribbean Island. In: (Talekar, N.S. ed.), Diamond back moth and other crucifer pests. Proc. 2nd Int. Workshop, 10-14 December, 1990, AVRDC Tainan, Taiwan, 233-243.

Arshad, Ali and Rana, K.S. 2012. A survey of aphidophagous lady bird beetles at some districts of Western Uttar Pradesh. Bionotes, 14: 21.

Choudhari, N., Ghosh, J. and Senapati, S.K. 2001. Incidence of insect pests of cabbage in relation to prevailing climatic conditions of Tarai region. Indian $J$. Entomol., 63(4): 421-428.

Cock, M.S.W. 1985. A review of biological control of pests in the commonwealth Caribbean and Bermuda upto 1992. Technical communication 9. Commonwealth Institute of Biological Control. Commonwealth Agricultural Bureaux, 218.

Gour, I.S. 2001. Impact of pesticidal application on natural enemies and pollinaters in mustard crop ecosystem. Dissertation. Ph. D. Thesis, Rajasthan Agricultural University, Bikaner.

Heathcoate, G.C. 1972. Evaluating aphid population on plants. In: Aphid Technology (Ed. H. V. Van Emden.) Academic Press, New York, pp.105-145.

Hugar, P.G., Anandhi, P., Varma, S. and Sarvanan, L. 2008. Seasonal incidence of important insect pests of mustard and their natural enemies in relation to weather parameters, in Allahabad region. J. Entomol. Res., 32(2): 109-112.

Joshi, B.G., Sitaramaiah, S., Satyanarayana, S.V.V. and Ramaprasad, G. 1979. Note on natural enemies of Spodoptera litura (Fab.) and Myzous persicae Sulz on fluecured tobacco in Andhra Pradesh. Curr. Sci., 45: 251-252.

Kitauchi, Y. And Nagami, T. 1984. Hymenopterous parasites of the cabbage moth Plutella xylostella L. Observed in cabbage fields of Oita prefecture. Proceeding of Association for Plant Protection, Kyushu, 30: 124-125.

Krishnamoorthy, A. and Mani, M. 1985. Investigations on Telenomus remus Nixon and Apanteles marginiventris Cresson against Spodoptera litura (Fab.) on cabbage. Entoman, 10: 277-280.

Kulkarni, A.V. and Patel, I.S. 2001. Seasonal incidence of mustard aphid (Lipaphis erysimi) and associated bioagent in Indian mustard (Brassica juncea) crop. Indian J. Agri. Sci., 71(10): 681-682.

Kumar, G., Anandhi, P., Varma S. and Elamathi, S. 2009. Seasonal occurrence of Brevicoryne brassicae and natural 
enemies on cabbage. Annals of Plant Protection Sci., 17(2): 476-478. 3.

Kumar, H. 1989. Distribution and abundance of aphids and their natural enemies in Southern-Eastern Rajasthn with special reference to chemical control of mustard aphid, Lipaphis erysimi (Kalt) M.Sc. (Ag.) Thesis, Rajasthan Agricultural University, Campus - Udaipur.

Kumawat, R.L. 2004. Predatory fauna associated with pest-complex of cabbage, their biology, multiplication and utilization in pest management. Ph.D. Thesis, Submitted to Rajasthan Agricultural University, Bikaner.

Liu, S.S., Wang, X.G. Shi, Z.H. and Guo, S.J. 2000. Biology of Oomyzus sokolowskii and effect of temperature on its population parameters. Acta Entomology Sinica, 43(2): 159-167.

Mandal, S.M.A. and Patnaik, N.C. 2008. Interspecific abundance and seasonal incidence of aphids and aphidophagous predators associated with cabbage. $J$. Biol. Control, 22(1): 195-198.

Mishra, S. 2003. Bio-ecology and management of mustard aphid, Lipaphis erysimi (Kalt.) on mustard with special reference to bio control agents. Ph.D. Thesis, Rajasthan Agricultural University, Bikaner, Campus: Jobner.

Noda, T., Miyasi, S., Yamada, S. and Konoshi, K. 1996. Larval and pupal parasitoids of diamondback moth Plutella xylostella (L.) in cabbage fields in Marioka Japan. Japanese J. Appl. Entomol. Zool., 40(2): 164-167.

Patra, S., Dhote, V.W., Alam, S.K.F., Das, B.C., Chatterjee, M.L. and Samanta, A. 2013. Population dynamics of major insect pests and their natural enemies on cabbage under new alluvial zone of West Bengal. The J. Plant Protection Sci., 5(1): 42-49.

Rao, S.R.K. and Lal, O.P. 2005. Seasonal incidence of mustard aphid, Lipaphis erysimi (Kalt) and diamondback moth, Plutella xylostella (L.) on cabbage. J. Insect Sci., 18(2): 106-110.

Singh, K.M., Singh, M.P. and Gupta, M.K. 2006. Seasonal incidence of mustard aphid, Lipaphis erysimi (Kalt.) and its natural enemies on radish in Arunachal Pradesh. J. Plant Protection and Environ., 3(1): 140-142. 7.

Singh, O.P. and Rawat, R.R. 1981. New record of Diaeretus rape M'Intosh. As an endoparasite of Lipaphis erysimi (Kalt.) from Madhya Pradesh. Sci. Culture, 48(8): 288-290.

Talekar, N.S. and Hu, W.J. 1996. Characteristics of parasitism of Plutella xylostella (Lepidoptera: Plutellidae) by Oomyzussoko lowskii (Hymenoptera: Eulphidae). Entomophaga, 41(1): 45-52.

Vekaria, M.V. 1998. Biology and Integrated management of Lipaphis erysimi Kalt. On mustard under North Gujarat conditions. $\mathrm{Ph}$. D thesis, Gujarat Agricultural University, S.K. Nagar., P. 61.

Vekaria, M.V. and Patel, G.M. 2005. Seasonal abundance of Lipaphis erysimi (Kalt.) and their natural enemies on important cultivars of mustard in North Gujarat. Indian J. Entomol., 67(4): 369-377.

Wang, X.G. Lui, S.S., He, J.H. and Guo, S.J. 1998. Investigations on parasitoids of diamondback moth in the suburban areas of Hangzhou. Acta Phytophylacica Sinica, 25(1): 20-26.

\section{How to cite this article:}

Jat, G.C., R. Swaminathan, P.C. Yadav, H.L. Deshwal, S.K. Dotasara, Suman Choudhary and Hari Singh Choudhary. 2017. Effect of Natural Enemies on the Population Dynamics of Insect-Pest of Cabbage Ecosystem. Int.J.Curr.Microbiol.App.Sci. 6(6): 696-708. doi: https://doi.org/10.20546/ijcmas.2017.606.082 